\title{
Multiple Distant Cutaneous Metastasis: An Uncommon Presentation of a Common Malignancy
}

\author{
Manjari Kishore ${ }^{1}$, Prajwala Gupta ${ }^{1 *}$, Purnima Malhotra ${ }^{1}$, Lalit Aggarwal ${ }^{2}$ and Minakshi Bhardwaj ${ }^{1}$ \\ 'Dept of Pathology, PGIMER, Dr RML Hospital, New Delhi \\ ${ }^{2}$ Dept. of Surgery, Lady Hardinge Medical College \& Hospital, New Delhi
}

\begin{abstract}
Cutaneous metastasis from carcinoma breast in areas outside the chest wall is very rare. We present a case of young female with bilateral breast swellings with a clinicoradiological diagnosis of Fibroadenoma wherein FNAC proved useful in establishing the diagnosis of bilateral breast carcinoma with distant cutaneous metastasis.
\end{abstract}

Keywords: Cutaneous, breast carcinoma, FNAC, metastasis.

\section{Introduction}

Breast carcinoma is commonly seen in women; however, cutaneous metastatic presentation is not often seen. ${ }^{[1]}$ Cutaneous metastasis from breast carcinoma is usually noted in areas near the primary tumor. We report a case of triple negative bilateral invasive ductal carcinoma breast with multiple distant metastatic cutaneous lesions over arm, trunk and thigh.

\section{Case Report}

A 36-year-old female presented to surgery out-patient department with complaints of fever, fatigue, backache and swelling in right breast for last 1 year and multiple swellings in left breast for last 2-3 weeks. The patient complained of multiple small cutaneous lesions over trunk, arm and thigh for last 1 month. The swelling in the right breast initially measured $1 \mathrm{X} 1 \mathrm{~cm}$ and had progressively increased to approximately 8 X10 cms. It was irregular, firm, non-tender with restricted mobility with few lobulations. Multiple swellings in the left breast showed rapid progression to present size, ranging in size from $1 \mathrm{X} 1$ to $2.5 \mathrm{X} 3 \mathrm{cms}$ [Fig 1a]. It was multinodular, firm, non-tender with restricted mobility. No ulceration, discharge or retraction of nipple was noted in both the breasts. The swellings were not fixed to the underlying chest wall or overlying skin.

On general examination, single right axillary lymph node was palpable, measuring $1 \mathrm{X} 1 \mathrm{~cm}$ and was firm, mobile and non-tender. Multiple cutaneous lesions were also found on right upper arm, right thigh, anterior abdominal wall and below the right breast [Fig 1a; arrow and Fig 1b]. These swellings measured approximately $1 \mathrm{X} 1 \mathrm{~cm}$ each and were firm to hard in consistency with bluish discoloration of the overlying skin.
Ultrasonography of bilateral breasts showed large lobulated irregular mass in right breast with multiple discrete nodules. Multiple heterogeneous nodules were noted in left breast. Few necrotic lymph nodes were seen in right axilla, largest measuring $1.2 \mathrm{X} 1 \mathrm{~cm}$. An impression of phyllodes tumor was given for right breast swellings and multiple fibroadenomas were suggested in left breast.

The hemogram parameters \& routine biochemical investigations were within normal limits. However, erythrocyte sedimentation rate (ESR) was found to be high (51 $\mathrm{mm}$ at the end of $1^{\text {st }}$ hour).

Multiple prick fine needle aspiration (FNA) was done from different sites of the large nodular bilateral breasts swellings and the right axillary lymph node. Geimsa \& Papanicolaou stained prepared from the bilateral breast swellings revealed high cellularity with presence of ductal cells showing moderate to marked pleomorphism and arranged in sheets, clusters, groups, occasional tubule formation and many singly scattered [Figure 2a]. These cells showed moderate anisonucleosis, coarse granular chromatin, some with prominent nucleoli, and irregular nuclear membrane. Background showed many lymphocytes, cystic macrophages and few stromal fragments with adhered malignant cells [Figure 2b]. Cytological features were consistent with bilateral ductal carcinoma breast. Smears from right axillary lymph node swelling showed tumor deposits along with foci of necrosis [Figure 2c].

FNA smears from the multiple subcutaneous swellings over right thigh, right arm, anterior abdominal wall revealed tumor deposits of malignant ductal epithelial cells as were seen in smears from breast swelling [Figure 2d]. 
FNA material for cell block was procured from the cutaneous swellings. Sections revealed Invasive ductal carcinoma [Figure 2e]. Trucut biopsy of bilateral breast showed Invasive ductal carcinoma (Not otherwise specified, NOS) with Nottingham grade III [Figure 2f]. Immunohistochemistry showed negative staining for ER, PR and Her-2-neu. Thus, it was a triple negative bilateral breast carcinoma.

Further, the ultrasonography study of abdomen revealed hepatosplenomegaly with multiple well defined hypoechoic metastatic lesions in both spleen and liver along with abdominal lymphadenopathy. Two-dimensional echocardiography showed normal study. Her chest X-ray and skeletal survey was also done and did not reveal any abnormality.

In view of disseminated presentation of bilateral breast carcinoma, a whole-body CT and PET scan was planned. However, patient refused and was referred to a higher oncology centre for further management.

\section{Discussion}

Cutaneous metastasis can be seen from various internal malignancies and sometimes can be the initial presentation of the underlying malignancy. ${ }^{[1-3]}$ The overall incidence of cutaneous metastasis from different malignancies is in the range of $0.7-10 \%{ }^{[3,4]}$. The most common malignancy metastasizing to skin is melanoma $(45 \%$ of all cutaneous metastasis cases) followed by breast carcinoma $(24.4 \%)$, nasal sinus $(20 \%)$, larynx $(16 \%)$, oral cavity $(12 \%){ }^{[1,3-4]}$

Cutaneous metastasis can present within few months to few years after diagnosis of the underlying malignancy. However, sometimes metastasis can be seen concurrently with the initial diagnosis of primary malignancy; as noted in our case. ${ }^{[2,3]}$

Though cutaneous metastasis from carcinoma breast is not so uncommon; distant metastasis is rarely noted. ${ }^{[4]}$ The usual site of involvement from breast carcinoma is head $\&$ neck region and areas in close proximity to the primary breast carcinoma. ${ }^{[2-4]}$ However, there are few reports of cutaneous metastasis from breast involving scalp, abdomen and upper extremities but only few cases have been noted in lower limbs. ${ }^{[4,5]}$ In our case, the patient had extensive cutaneous involvement which was also seen at distant sites like in upper arm and right thigh; thigh being a very rare site. Navaratnam et al ${ }^{[5]}$ reported a similar case of cutaneous lobular breast carcinoma metastasis to her anterior right leg. To the best of our knowledge, no case with metastasis to thigh has been reported; index case being the first.

Clinically, different patterns of cutaneous metastasis from breast carcinoma are noted. These are nodular, telangiectatic, inflammatory, sclerotic, alopecia, zosteriform, papulovesicular, ulcerative, annular erythema and paget's disease. ${ }^{[3,4-7]}$ Nodule is the most common

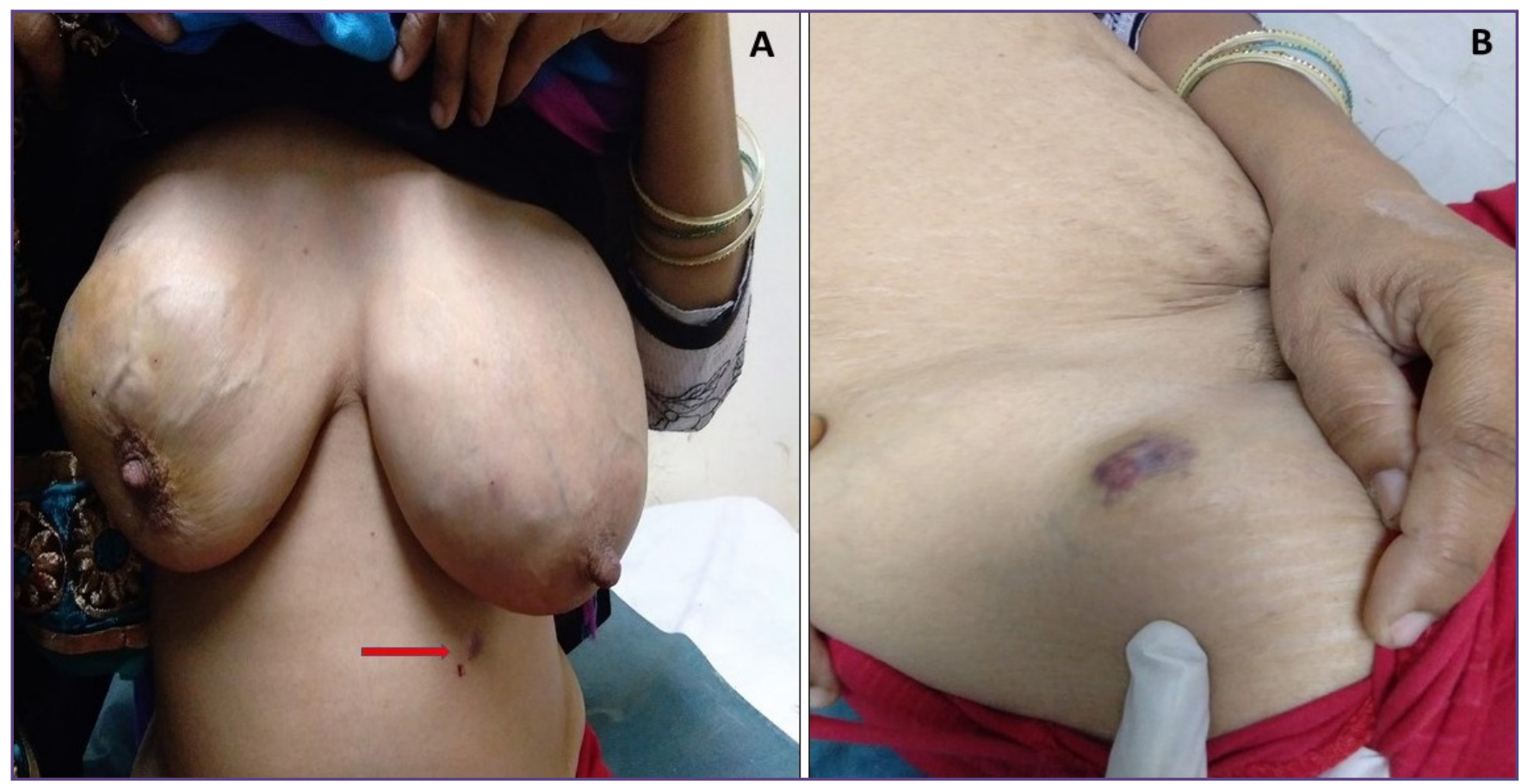

Fig. 1: A-Bilateral breasts with large irregular nodular swellings. A small pigmented cutaneous nodule in left upper abdominal wall, below the left breast (arrow). B- Cutaneous pigmented nodular swelling on upper thigh. 

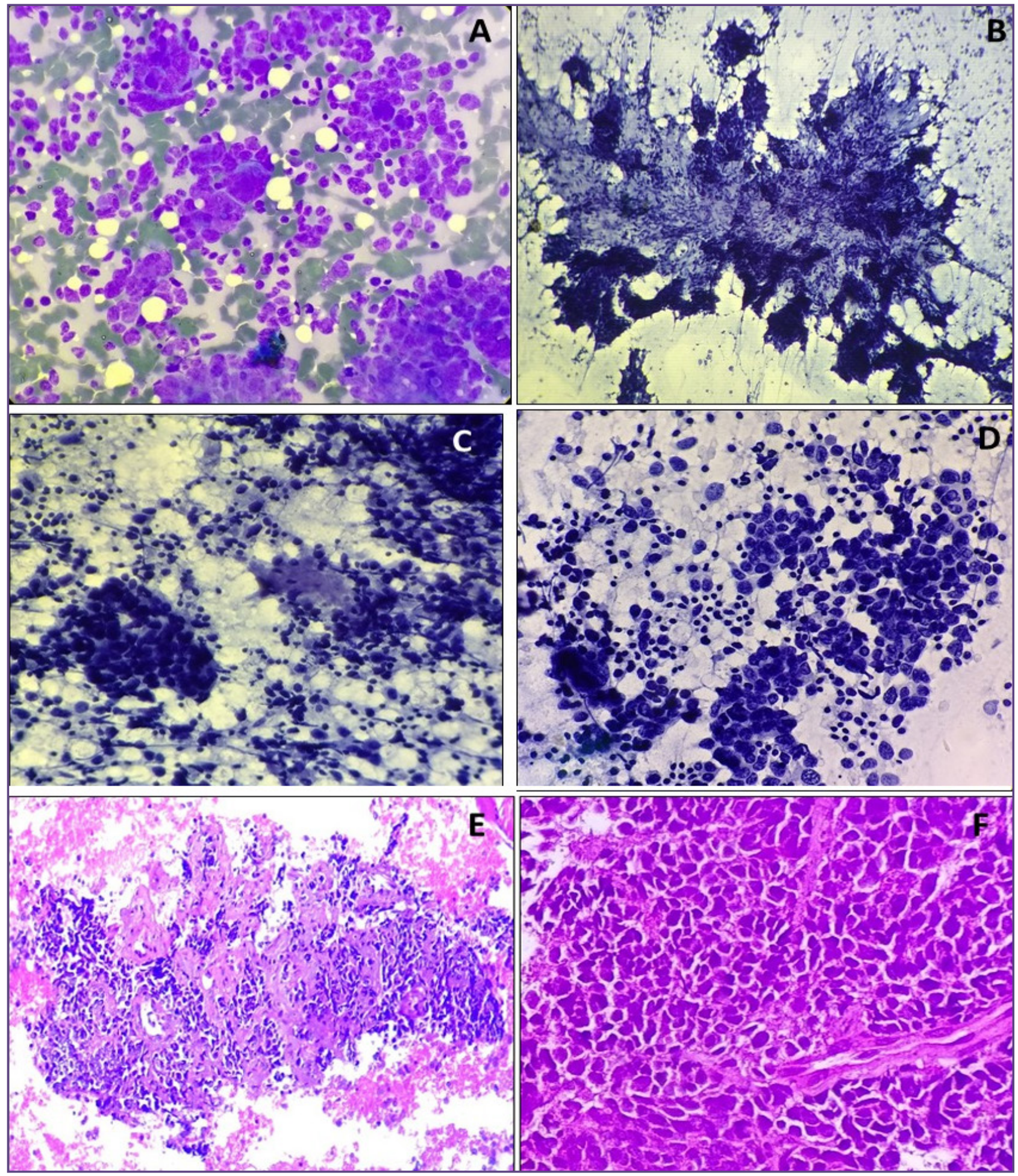

Fig. 2: A- FNA smear from breast showing malignant ductal cells with occasional bizarre form [Giemsa, X400]. B- Large stromal fragment with infiltrating malignant cells [Pap, X200]. C- Smear from axillary lymph node shows metastatic malignant epithelial cells with foci of necrosis [Pap, X400]. D- Cellular smears from cutaneous metastatic lesion revealing metastatic deposits of tumor cells [Pap, X400]. E- Cell block sections from thigh swelling shows malignant ductal cells [H\&E, X200] .F- Trucut biopsy section from breast shows cord-like infiltration of malignant cells [H\&E, X200].. 
presentation of metastasis and zosteriform pattern being least. ${ }^{[3,5]}$

Triple negative breast cancer (TNBC) represents a unique subgroup with a specific molecular profile, lacking the expression of gene for ER, PR or Her-2-neu. ${ }^{[8]}$ They present as a larger sized tumor, grade 3, pushing margins, development of recurrence, distant metastasis and poorer Nottingham Prognostic Index. Usually noted in younger individuals with BRCA-1 mutation, TNBC is also termed as basal-like. ${ }^{[9]}$ Histologically, TNBC may show specific morphologic types such as secretory cell carcinoma/ adenoid cystic carcinoma, medullary carcinoma with prominent lymphocyte infiltrate and grade 3 invasive ductal carcinoma with no specific subtype.

Triple negative cases may metastasize even when small, frequently to viscera, and brain. They have an aggressive behaviour pattern, a relative lack of effective therapies $\&$ a poor prognosis ${ }^{[8,9]}$. However, approximately $30 \%$ completely respond to chemotherapy. The standard treatment is chemotherapy and radiotherapy in cases with widespread metastasis. However, there is higher risk of early recurrence and dismal survival with metastasis ${ }^{[8,9]}$. Currently various newer modalities are being developed like anti-angiogenic drugs, mTOR inhibitors and EGFR inhibitor ${ }^{[8,9]}$. Cetuximab is a chimeric monoclonal antibody that inhibits the EGFR.

\section{Conclusion}

Cutaneous metastasis from breast carcinoma is common in women. However, distant cutaneous metastasis to areas outside chest and trunk is very rare; such as thigh deposit in our case. Metastatic cutaneous deposits may be the initial presentation in a clinically unsuspected case of triple negative breast carcinoma and FNAC with cell block helps in prompt diagnosis.

\section{References}

1. Nashan D, Muller M.L. Cutaneous metastases of visceral tumours: a review. J Cancer Res Clin Oncol. 2009; 135: $1-14$.

2. Hu S.C., Chen G.S. Cutaneous metastases from different internal malignancies: a clinical and prognostic appraisal. J Eur Acad Dermatol Venereol. 2008; 22: 735-40.

3. Mahore SD, Bothale KA, Patrikar AD, Joshi AM. Carcinoma en cuirasse: a rare presentation of breast cancer. Indian $\mathrm{J}$ Pathol Microbiol. 2010;53:351-8.

4. Dobson CM, Tagor V, Myint AS, Memon A. Telangiectatic metastatic breast carcinoma in face and scalp mimicking cutaneous angiosarcoma. J Am Acad Dermatol. 2003; 48: 635-6.

5. Navaratnam AV, Chandrasekharan S. Remote Cutaneous Breast Carcinoma Metastasis Mimicking Dermatitis. Indian J Dermatol. 2015;60:106-8.

6. Conner KB, Cohen PR. Cutaneous metastasis of breast carcinoma presenting as alopecia neoplastica. South Med J 2009; 102: 385-9.

7. Afrose R, Akram M, Siddiqui SA. Papular skin lesions: Clue to a recurrence of breast cancer on fine needle non-aspiration cytology (FNNAC). J Cytol 2015;32:68-70.

8. Ueno NT, Zhang D. Targeting EGFR in Triple Negative Breast Cancer. J Cancer 2011; 2:324-328.

9. Park HS, Jang MH, Kim EJ, Kim HJ, Lee HJ. Et al. High EGFR gene copy number predicts poor outcome in triplenegative breast cancer. Modern Pathology 2014;27:1212-22.

*Corresponding author:

Dr. Prajwala Gupta, Associate Professor, Dept of Pathology, Room No.302, 3rd floor, OPD building, Dr RML Hospital, Baba Khadak Singh Maarg

New Delhi-110001, India

Phone: +91 991036227

Email: prajwala2000@yahoo.com

Financial or other Competing Interests: None. 